\title{
APT PC and APT II: Experiment development systems for the IBM PC and Apple II
}

\author{
STEVEN E. POLTROCK and GREGORY S. FOLTZ
}

\begin{abstract}
Systems for creating and running psychology experiments on an Apple II or IBM PC are described. Without programming, psychologists using the APT systems can construct and con. duct an experiment, summarize the data, and revise the experiment. The systems are easily used by students but powerful enough to be used by psychologists in their research.
\end{abstract}

The APT system allows psychologists to create new experiments, edit existing experiments, run experiments, and perform preliminary data analyses without any programming. The original version of APT (Poltrock \& Foltz, 1982) was intended to simplify creating, modifying, and running psychometric and cognitive tests and experiments. APT II and APT PC expand the capabilities of the original version and are much easier to use. Using an interactive editor, experiments are created and modified by first defining different kinds of stimuli, then entering the text of each stimulus. Running an experiment generally requires only providing the names of input and output files. Summarizing the results requires little more than defining an experimental condition for each stimulus.

Psychologists who use the APT systems can concentrate on the details of their experimental methodology without being distracted by the programming required to implement the methodology. The APT systems were designed to be used in research, but have also proven useful for teaching laboratory methods. Instructors have used APT to construct experiments that demonstrate cognitive phenomena and experimental methodology. Because the APT systems are easy to use, students can construct their own experiments or explore methodological variations by editing an existing experiment.

Using the APT systems, psychologists avoid programming, but at the cost of constraints on their experiments. Stimuli can be composed only of text; no graphics, animation, sounds, or external devices are supported. Stimuli and responses are synchronized such that no stimulus can be presented until the response to the preceding stimulus is completed. Consequently, double stimulation experiments, which require simultaneous timing of multiple responses, cannot be conducted. The stimulus order cannot be contingent on the responses, which rules out use of the APT systems for computer assisted instruction.

\footnotetext{
These systems were developed and are sold by the partnership of Gregory Foltz and Steven Poltrock. The cost of either system is $\$ 100$, and the source code for either system costs an additional $\$ 100$. To purchase APT II or APT PC, to request reprints of this article, or to request additional information, write to either of the authors at their home addresses. Steven Poltrock's address is 8200 Neely Dr. \#149, Austin, TX 78759. Gregory Foltz's address is 1650 Fairoak Dr., Colorado Springs, CO 80907.
}

There are also timing and size limitations, which are described below. Despite these constraints, the vast majority of memory and cognition experiments can be conducted using the APT systems.

\section{HARDWARE AND SOFTWARE REQUIREMENTS}

\section{APT II}

The names of the two APT systems are intended to suggest the fundamental difference in their hardware and software requirements. APT II requires an Apple II, Apple II Plus, or Apple IIe with 64K RAM and either a Mountain Hardware Clock or a Timemaster II H.O. clock. If a Mountain Hardware Clock is used, it must be modified so that interrupts occur every millisecond, as described in the APT II manual. The Apple Ile has several advantages over its predecessors, the Apple II and Apple II Plus, including a keyboard and display that support upperand lowercase characters. The Apple Ile also provides a signal that indicates when the display refresh cycle starts, allowing substantially better precision for response times and display durations. The same precision can be achieved with the Apple II or Apple II Plus, however, by making a simple hardware modification described in the APT II manual. This modification is not required if less precise timing is acceptable. APT II is written in Apple Pascal, with time-critical portions written in 6502 assembly code. The Apple Pascal operating system is required to use APT II.

\section{APT PC}

APT PC requires an IBM PC (AT or XT) or one of the many PC clones with 256K RAM. Parts of APT PC do not run on certain clones (e.g., the Sperry PC) that lack the standard internal clock, because assembly language procedures in APT PC use the clock to time responses and display durations. The display can be driven either by a monochrome or graphics display adapter. With a monochrome adapter, each display line contains 80 characters, whereas the graphic adapters allow either $\mathbf{4 0}$ or 80 characters per line. When an experiment is created or edited, the experimenter is queried regarding the desired line length, and APT PC sets the appropriate mode 
of the graphic display adapter. The graphic adapter also provides a signal indicating the start of each display refresh cycle, which allows more precise timing of responses and display durations when experiments are run with this configuration. APT PC is written in Turbo Pascal, with time-critical portions written in assembly language. A DOS operating system, Version 2.0 or later, is required to use APT PC.

\section{CREATING AND EDITING EXPERIMENTS}

Experiments are created and modified using MAKEXP, an interactive experiment editor. Psychologists can create an experiment using MAKEXP, test run the experiment, then use MAKEXP to edit the instructions, practice trials, procedure, and stimuli. Nearly every aspect of an experiment is modifiable.

\section{Items, Item Types, and Global Parameters}

A trial is typically the basic unit of psychology experiments, but each trial may require presentation of a sequence of separate stimuli. In the APT systems, the basic unit is an item, and a trial consists of one or more items. Each display of text on the screen requires a separate item (except for accuracy or response time feedback). The investigator defines item types that characterize how items of that type are displayed, whether any response is expected, whether feedback should be presented, and whether response information should be recorded in the output file. In addition to defining items and item types, the investigator sets the values of some global parameters that affect all items. For example, one global parameter is the duration of feedback messages, and another determines whether the total number of correct responses is reported at the end of the experiment. An experiment is constructed by specifying items, item types, and global parameters. The information required for each of these three components is summarized in Table 1 and described below.

Consider how a replication of one of Sternberg's (1969) studies of short-term memory scanning may be constructed. The experiment may begin with instructions, followed by several practice trials. Then the subject may be given an opportunity to repeat the instructions and practice trials or begin the experiment. Using the varied-set procedure each trial of the experiment would consist of three items or presentations: a memory set followed by a warning stimulus followed by a test stimulus. The order of the trials should be randomized for each subject, but of course the instructions cannot be randomized. Furthermore, the order of the items that comprise a trial cannot be randomized. The following sections describe how the items, item types, and global parameters would be defined to replicate Sternberg's study.

\section{Defining Item Types}

To construct this experiment, an investigator would first define six item types: (1) the instructions, (2) the prac-
Table 1

The Information Required for the Three Components of an Experiment
Item Types
1. Name of the item type
2. Event that terminates items
3. Item feedback (RT and/or accuracy)
5. Position of the item text
Items
1. Item type
2. Text presented to the subject
3. Answer
4. Randomization code
Global Parameters
1. Fixed or random item order
2. Fixed or random item set order
3. Fixed or random block order
4. Interval between items
5. Duration of feedback
6. Legal response set
7. Whether to show number correct

4. Information to be recorded (RT and/or accuracy)

tice trials, (3) the choice of continuing or repeating the instructions and practice trials, (4) the memory set, (5) the warning stimulus, and (6) the test stimulus. The investigator is asked to name each item type, and these names are used to construct a menu of all available item types. After naming an item type, the investigator defines how all items of this type are terminated. Instructions could be terminated when the subject presses any key; the memory set and warning stimulus could be terminated after a duration defined by the experimenter. The test stimulus could be terminated when the subject presses either of two keys, defined as the legal response set; any other response would cause an error message. The subject can be offered a choice of continuing the experiment or repeating the instructions and practice trials through use of an option that restarts the experiment if the " $y$ " key is pressed while an item of this type is displayed.

There are 10 alternative ways to terminate a display; the investigator simply selects the desired option from a menu. In addition to those already described, an item can be terminated by a series of typed characters ended by the RETURN key (a string response), by a contact closure obtained through a game input device (e.g., a joystick), or by the earlier of a response or an elapsed time. The duration of a display can be defined either in milliseconds or display refresh cycles $(16.67 \mathrm{msec}$ in countries with $60 \mathrm{~Hz}$ power; $20 \mathrm{msec}$ in countries with $50 \mathrm{~Hz}$ power). To support simple masking experiments, one option presents an item for a fixed number of refresh cycles, then displays the next item with an interitem interval of $0 \mathrm{msec}$.

After selecting a method for terminating items, the investigator specifies whether feedback follows these items. Four alternatives are presented in a menu; feedback regarding accuracy, response time, both, or neither may be presented. The feedback consists of the word CORRECT or WRONG and/or TIME = (followed by the response time in milliseconds). In the memory-scanning ex- 
periment, feedback would be appropriate only for the practice trials and the test stimuli, not for the instructions, memory set, or warning stimuli. Even if feedback is not provided to subjects, the investigator may select accuracy feedback for the practice trials and test stimuli when first constructing the experiment. This feedback enables the investigator to test that the correct answer was entered for each stimulus. Later, the item type can easily be edited to eliminate this feedback.

Another menu lists the options for recording response information in the output file. The response, the response time, both, or neither may be recorded. In this memoryscanning experiment, no information would be recorded for all item types except the test stimulus, and both response and response time would be recorded for the test stimulus.

The last menu allows the investigator to specify the position of the item text on the display. Both the line and the column in which the item text begins can be specified. An alternative to specifying a starting column is to center each line horizontally. Typically, none of these options would be used for the instructions item type, but for the memory set, warning stimulus, test stimulus, and practice trials, the investigator could choose to center each line and specify a starting line number.

\section{Entering Items}

After defining all six item types, the investigator enters the items. (At least one item type must be defined before any items are entered, but new item types can be added at any time.) As Table 1 shows, four kinds of information must be specified for each item. The information required to construct the first 14 items of the memoryscanning experiment is shown in Table 2.

For each item, the investigator first selects the item type from a menu of the names supplied by the investigator when the item types were defined. The first item should be the first screen of instructions. After selecting "instructions" from the menu of item types, the investigator types the text of the item (i.e., the actual instructions). APT PC provides screen editing capabilities that allow the investigator to position the cursor anywhere on the screen and use the delete and backspace keys to correct errors in the item text. The end of the item is denoted by typing the \# symbol.

In addition to the item type and text, the investigator must supply an answer and randomization code for each item. For those items that do not have a correct answer, the investigator presses a space bar. Consequently, the space bar can never be used as a correct response. The answer is used to score each response.

The randomization code is used to group items and determine their order of presentation to the subject. The investigator should assign 0 as the randomization code for the instructions, the practice trials, and the item that asks the subject to decide whether to repeat the instructions and practice trials. All items with a randomization code of 0 are presented in the same order in which they were
Table 2

Information Required for the First 14 Items of a Varied Set Memory-Scanning Experiment

\begin{tabular}{|c|c|c|c|c|}
\hline \multicolumn{2}{|c|}{ Item Type } & \multicolumn{3}{|c|}{ Randomization } \\
\hline Item & Name & Content & Code & Answer \\
\hline 1 & instructions & explanation of procedure & 0 & space \\
\hline 2 & memory set & 715 & 0 & space \\
\hline 3 & warning & $*$ & 0 & space \\
\hline 4 & practice & 1 & 0 & $y$ \\
\hline 5 & memory set & 28 & 0 & space \\
\hline 6 & warning & * & 0 & space \\
\hline 7 & practice & 4 & 0 & n \\
\hline 8 & repeat & explanation of options & 0 & space \\
\hline 9 & memory set & 2 & A & space \\
\hline 10 & warning & $*$ & A & space \\
\hline 11 & test stimulus & 5 & A & n \\
\hline 12 & memory set & 4135 & B & space \\
\hline 13 & warning & $*$ & B & space \\
\hline 14 & test stimulus & 3 & B & $y$ \\
\hline
\end{tabular}

entered. Consequently, the investigator must enter these items in the order they are to be presented.

The memory set, warning stimulus, and test stimulus for each trial must be entered in their presentation order and assigned the same randomization code (any letter or number). Successive items with the same randomization code form an item set. The investigator can choose to randomize the order of item sets, thereby randomizing the order of trials, without randomizing the order of the items within a set.

Note that trials are not defined in the APT systems. The investigator defines collections of items that correspond to trials by appropriate assignments of randomization codes. The memory-scanning experiment only requires one level of grouping, but a second level is available. Item sets can be grouped into blocks. A block is defined as all items between two items with codes equal to zero, or all items between one item with a code equal to zero and the beginning or end of the experiment. The investigator can choose to randomize or leave unchanged the order of items within item sets, item sets within blocks, and blocks within the experiment. The block order can also be specified when the experiment is run to counterbalance a small number of blocks.

These three hierarchical levels and the accompanying choices regarding randomization are the means of controlling item order (see Poltrock \& Foltz, 1982, for further details). As an example of their effectiveness, a reading comprehension experiment can be conducted in which each of several passages is followed by a series of questions. The order of both the passages and the questions can be randomized, yet each passage would still be followed by the correct set of questions. Similarly, the replication of Sternberg's (1969) experiment can be transformed into a fixed-set procedure by presenting a long series of warning stimuli and test stimuli after each memory set.

The order in which the items are entered is critically important. They must be entered in an order that would appear sensible if presented to a subject. If the inves- 
tigator first entered all the memory sets, then all the warning stimuli, and finally all the test stimuli, there would be no way to reorder the items and reconstruct the intended trial structure. Each memory set, warning stimulus, and test stimulus must be entered as three successive items. The task of entering all these items is somewhat simplified by an option that allows a previous item to be copied. Because the warning stimulus items have the same item type, text, and answer, they can be entered by copying a previous warning stimulus. The investigator is asked to provide only the randomization code when items are copied.

\section{Setting Global Parameters}

Seven global parameters determine characteristics of the procedure that are applicable to all item types. When the global parameters are set, MAKEXP presents a description of each parameter and ensures that the investigator's response is within the range of acceptable values. Three of the parameters determine the presentation order of the items. These parameters specify whether the items, item sets, and blocks are randomized or presented in the order they were entered. For the memory-scanning experiment, item sets correspond to trials consisting of a memory set, warning, and test stimulus. Consequently, randomizing item sets corresponds to randomizing trials. Items should not be randomized, however, because that would rearrange the order of the components of a trial. Randomizing block order is not meaningful because the experiment contains only one block bounded by the beginning and end of the experiment.

Two other parameters specify time intervals: One parameter specifies the interval between successive items, and the other specifies the duration of the accuracy and response time feedback messages. The sixth parameter defines the legal response set, which governs the acceptable responses to items terminated by a legal response. For example, the legal response set would be "yn" if subjects were intended to respond by typing either $\mathbf{y}$ or $\mathbf{n}$. The last parameter indicates whether the total number of correct responses should be presented to the subject at the end of the experiment.

\section{Reviewing and Editing Experiments}

Needless to say, errors will occur when entering the item types, items, and global parameters. MAKEXP enables the investigator to review and correct all three components of an experiment. When reviewing item types, each menu is displayed with the investigator's choices indicated. The original choices can be accepted or modified.

When reviewing and editing items, each item is referenced by its ordinal number. The item type, item text, answer, and randomization code are displayed and can be changed for the selected item. APT PC presents the item text exactly as it will appear in the experiment, adjusted to the starting line and either centered or adjusted to the starting column. If the item text is changed, APT II requires that the entire text be typed anew, whereas
APT PC provides a screen editor for modifying the text. It has been noted that the order of items is important. When reviewing and editing items, the order can be modified by inserting an item in front of an existing item or by deleting an item.

When reviewing global parameters, the original value of each parameter is displayed, and the investigator is asked to enter a new value.

\section{Programmatic Experiment Construction}

The output of MAKEXP is a file containing the global parameters, item type definition, and the content of each item. The format of this file is described in the APT manuals. Some investigators may prefer to write programs that generate this file rather than type the text of every item. This approach is certainly appropriate when there is a large number of items that can be generated algorithmically. After generating all the items, MAKEXP could be used to edit the item types and global parameters, and to enter instructions and practice trials.

\section{RUNNING EXPERIMENTS}

Experiments are conducted using the RUNEXP program. Generally, the only information that RUNEXP requires from the experimenter is the name of an input file generated by MAKEXP and the name of an output file for the results of the experiment. If, however, block order is to be permuted, RUNEXP asks whether the experimenter intends to specify the block order or prefers that block order be randomized.

The output file produced by RUNEXP contains five columns of information, with each row corresponding to an item for which the response or response time was recorded. The columns contain the item number, its presentation order, the accuracy of the response, the response time, and the actual response.

\section{ANALYZING THE RESULTS}

\section{Demonstration Experiments}

Three experiments provided with the APT systems demonstrate how to use their features. New experiments can be created by copying these experiments and editing the item types, global parameters, and items. For two of these experiments, analysis programs written in Pascal demonstrate how to summarize the results of an experiment. These programs simply compute the mean response time and the number of correct responses for each condition.

\section{Subject and Item Analyses}

APT PC includes two additional analysis programs. ITEMANAL computes summary statistics for each item averaging over subjects. SUBJANAL computes summary statistics for each subject, averaging across items within the same condition. When using SUBJANAL, the investigator is asked to specify an experimental condition for each item. SUBJANAL reports the number of correct, 
incorrect, and unscored responses for each condition. It also reports the mean and standard deviation of response times for each kind of response in each condition.

\section{TIMING AND SIZE LIMITATIONS}

\section{Stimulus Onset Time}

The accuracy and precision of stimulus onset time is affected by whether a signal indicating the start of the video refresh signal is available. If this signal is available, then APT executes the instruction to display the stimulus just as the video refresh starts at the upper left corner of the screen. The actual time of the stimulus onset depends on the position of the stimulus on the screen, but can be estimated as $2 / 3$ of a millisecond for each line of text. If the signal is not available, then the stimulus onset begins at a time uniformly distributed between 0 and $16.67 \mathrm{msec}$ ( $20 \mathrm{msec}$ where $50 \mathrm{~Hz}$ current is used) after APT executes the instruction to display it.

\section{Minimum Interitem Interval}

During the interval between items, the APT systems perform a variety of functions. The response, if any, must be interpreted, scored, and saved; the time must be read and saved; and the text of the next item must be written into screen memory. The minimum possible time between successive items depends on exactly which functions are performed. For APT II, the minimum time is approximately $77 \mathrm{msec}$ plus $6 \mathrm{msec}$ for each line of text. For APT PC, the minimum time is approximately $22 \mathrm{msec}$ plus $16.7 \mathrm{msec}$ for each line of text. More precise formulas are provided in the manuals.

\section{Size Limitations}

The maximum number of items in an experiment is 1,200 for APT II and 5,000 for APT PC. The maximum number of characters used to describe an experiment is 20,000 for APT II and 32,767 for APT PC.

\section{CONCLUSION}

Several other systems have been developed to create and run psychology experiments. CEDATS (Eamon,
1982) allows a student to create new experiments by describing the experimental design, the stimuli, and the type of experiment. It supports categorization, same-different, and study-test experiments. Like the APT systems, CEDATS requires no programming. It also allows the student to think in terms of experimental paradigms and designs, but it restricts the student or researcher to the three programmed paradigms. A far greater range of experiments can be created using the APT systems.

Other systems require programming to create an experiment. The Cognitive Testing Station developed by Digitry, Inc., includes expensive hardware and a library of procedures and functions. Psychologists must create experiments by writing Pascal programs. M.T.S. (Foree, Eckerman, \& Elliott, 1984) provides a collection of tasks, but programming is required to create new experiments and additional hardware is required.

The APT systems support creation of new experiments without the need to program, and consequently can be used in both research and teaching laboratories. Simultaneously, the APT systems provide some advantages that are normally obtained only by programming an experiment. Psychologists are not constrained to a preprogrammed set of paradigms, but can create their own paradigms. Furthermore, psychologists can iteratively create, test, and edit experiments. An existing experiment can be copied and edited to create methodological variations. Stimuli for an experiment can even be generated by programming, then edited.

\section{REFERENCES}

EAMON, D. B. (1982). CEDATS: A cognitive experimental design and testing system. Behavior Research Methods \& Instrumentation, 14, 142-145.

Foree, D. D., Eckerman, D. A., \& Eluotr, S. L. (1984). M.T.S.: An adaptable microcomputer-based testing system. Behavior Research Methods, Instruments, \& Computers, 16, 223-229.

Poltrock, S. E., \& Foltz, G. S. (1982). An experimental psychology laboratory system for the Apple II microcomputer. Behavior Research Methods \& Instrumentation, 14, 103-108.

STERNBERG, S. (1969). Memory-scanning: Mental processes revealed by reaction-time experiments. American Scientist, 57, 421-457. 